Research Paper

\title{
Direct Reprogramming of Human Amniotic Fluid Stem Cells by OCT4 and Application in Repairing of Cerebral Ischemia Damage
}

\author{
Mingde Qin ${ }^{1 *}$, Ruihua Chen ${ }^{2 *}$, Hong Li ${ }^{3}$, Hansi Liang ${ }^{1}$, Qun Xue ${ }^{2}$, Fang $\mathrm{Li}^{1}$, Ying Chen ${ }^{3}$, Xueguang \\ Zhang $^{1,2}{ }^{凶}$ \\ 1. The Stem Cell and Biomedical Material Key Laboratory of Jiangsu Province (the State Key Laboratory Incubation Base), Suzhou, Jiangsu Province, \\ P.R.China. \\ 2. Jiangsu Institute of Clinical Immunology, The First Affiliated Hospital of Soochow University, P.R.China. \\ 3. The Reproductive and Genetic Center, the Municipal Hospital of Suzhou, Suzhou, Jiangsu Province, P.R.China. \\ ${ }^{*}$ Co-First authors.
}

$\square$ Corresponding author: Professor Xueguang Zhang, M.D., Ph.D. The Stem Cell and Biomedical Material Key Laboratory of Jiangsu Province (the State Key Laboratory Incubation Base), Suzhou, Jiangsu Province, P.R.China. Jiangsu Institute of Clinical Immunology, The First Affiliated Hospital of Soochow University, P.R.China.708 RenMin Road. Soochow, P.R.China. Telephone: 86-512-65732002, e-mail: xueguangzh@126.com.

(c) Ivyspring International Publisher. Reproduction is permitted for personal, noncommercial use, provided that the article is in whole, unmodified, and properly cited. See http://ivyspring.com/terms for terms and conditions.

Received: 2014.11.13; Accepted: 2016.02.09; Published: 2016.03.11

\begin{abstract}
Amniotic fluid stem cells (AFSCs) are a type of fetal stem cell whose stemness encompasses both embryonic and adult stem cells, suggesting that they may be easily and efficiently reprogrammed into induced pluripotent stem cells (iPSCs). To further simplify the reprogramming process, the creation of AFSC-derived iPSCs using a single factor is desirable. Here we report the generation of one-factor human AFSC-iPSCs (AiPSCs) from human AFSCs by ectopic expression of the transcription factor OCT4. Just like human embryonic stem cells, AiPSCs exhibited similar epigenetic status, global gene expression profiles, teratoma formation and in vitro \& in vivo pluripotency. Our results indicate that the OCT4 is necessary and sufficient to directly reprogram human AFSCs into pluripotent AiPSCs. Moreover, reflecting the similar memory characteristics of AFSCs and neural stem cells, we show that AiPSC membrane-derived vesicles (MVs) repair cerebral ischemia damage. We anticipate that the successful generation of one-factor AiPSCs will facilitate the creation of patient-specific pluripotent stem cells without the need for transgenic expression of oncogenes. Moreover, MVs from tissue-specific AiPSCs have potential in tissue repair, representing a novel application of iPSCs.
\end{abstract}

Key words: Induced pluripotent stem cell (iPSCs), Amniotic fluid stem cell (AFSCs) \& Membrane-derived vesicle (MV).

\section{Introduction}

The generation of induced pluripotent stem (iPSCs) cells from mouse [1] and human [2] somatic cells by epigenetic expression of defined factors has potential application for generating patient-specific iPSCs and stem cell treatment [1-4]. The molecular mechanisms underlying iPSC reprogramming remain incompletely understood due to several variables within the system, such as the number of exogenous factors required and the heterogeneity of target cells.
Given that reactivation of the $M y c$ oncogene in mice frequently causes tumours in chimeras and offspring derived from iPSCs [4], alternative approaches are required for iPSC generation that will avoid the use of oncogenes as transgenes and use the least factors. For example, one-factor iPSCs have been generated by overexpression of OCT4 only in both mouse [6] and human [7] neural stem cells (NSCs). NSC derived iPSCs generated from Human NSCs are normally 
isolated from fetal brain tissue however, the limited supply limits the clinical application of one-factor iPSCs derived from human NSCs.

Amniotic fluid is known to contain multiple cell types derived from the developing fetus, some of which can be reprogrammed into iPSCs. For example, IPSCs have been generated from human amniotic fluid-derived cells using four-factor [8] and two-factor (OCT4 and SOX2) [9] reprogramming systems without the use of oncogenes. A more recent report has described the isolation of a novel type of human amniotic fluid stem cells (AFSCs) [10], which represent $1 \%$ of the population of cells obtained from amniocentesis, and whose stemness encompasses both embryonic stem cells (ESCs) and adult stem cells. AFSCs are multipotent, and are characterized by expression of the receptor for stem cell factor c-Kit (CD117), in addition to OCT4, SOX2, NANOG and SSEA-4. These data led us to consider the possibility of using AFSCs to generate amniotic stem cell-derived one-factor human iPSCs, or AiPSCs.

Membrane-derived vesicles (MV)s are small vesicles released from the plasma membrane that, depending upon their cell type of origin, possess the capacity to repair local tissue damage and to modulate local tissue processes, such as angiogenesis [11-14]. Embryonic stem (ES) cells are a rich source of MVs and ES-derived MVs contain a variety of stem cell-specific molecules that modulate the growth of target cells and contribute to cell-fate decision [15]. Accordingly, given the similarity in memory characteristics between AFSCs and NSCs, we hypothesized that MVs isolated from AiPSCs would support both expansion of pluripotent stem cells, and mediate the repair of neuronal damage.

In this study, we describe the generation from human AFSCs of four one-factor (OCT4) human AiPSC cell lines (1F AiPSCs) and 18 two-factor (OCT4 \& SOX2) human AiPSC cell lines (2F AiPSCs). We found that the majority of these cell lines maintained a normal karyotype after propagation through up to 35 passages, and expressed a complement of pluripotency markers similar to that of embryonic stem cells. Moreover, the cells were efficiently differentiated into all three germ layer cells in vitro, and were found to be capable of teratoma formation in vivo. Finally MVs isolated from AiPSCs were used to repair cerebral ischemia damage and reduce infarct area relative to control animals. Our results indicate that OCT4 is necessary and sufficient to directly induce pluripotency in AFSCs, and that MVs derived from AiPSCs have therapeutic potential in the treatment of cerebrovascular disorders.

\section{Materials and Methods}

\section{Culture and Inactivation of mouse embryonic and human foreskin fibroblasts}

Mouse embryonic fibroblasts (MEF) were isolated from BALB/c mice obtained from the Chinese Academy of Sciences, at 13 or 14 d.p.c. (day post-coitum). MEFs and human foreskin fibroblasts were grown on T-75 flask in DMEM (Gibco, Rockville, MD) containing $10 \%$ fetal bovine serum (FBS), $1 \%$ glutamine and 1\% penicillin-streptomycin (Gibco), at $370 \mathrm{C}$ under a 5\% CO2 atmosphere. For inactivation, MEF was incubated with $10 \mu \mathrm{g} / \mathrm{ml}$ of mitomycin C containing medium for 2 hour at $37^{\circ} \mathrm{C}$. Initiative MEF was plated at a density of 50,000 cells $/ \mathrm{cm} 2$ on 6 -well plate or $100 \mathrm{~mm}$ culture dish and readied for use.

\section{Isolation and Culture of human AFSCs and human ESC}

Human AFSCs were isolated from human amniotic fluid (19-21 week gestation). Human AFSCs samples collection with written consent of subjects and the written approval from the Ethical Review Board. All experimental procedure using Human AFSCs samples in this study was reviewed and approved by the ethics committee.

Each time, $5 \mathrm{ml}$ of human amniotic fluid was used for isolation of human AFSCs. After PBS washing and centrifuge, the cells were on Petri dishes in human AFSC culture medium: a-MEM medium (Gibco, Rockville, MD) containing 15\% fetal bovine serum (FBS) (Gibco), 1\% glutamine and 1\% penicillin/streptomycin (Gibco), supplemented with 18\% Chang B and 2\% Chang C (Irvine Scientific Santa Ana, CA) at $370 \mathrm{C}$ under a 5\% CO2 atmosphere. After initial 5-7 days, small amount of cells were attached then the attached cell expanded for 5 days ready for immunoselection by c-Kit. For immunoselection of c-Kit-positive human AFSCs, after Trysin-EDTA digestion, the cells were selected with monoclonal anti-CD117 directly conjugated to MicroBeads (Miltenyi Biotec, Auburn, CA). Human AFSCs were subcultured routinely at a dilution of 1:5 and propagated at about $70 \%$ of confluence for every $2-3$ days.

The human ESC cell line ShhES2, a kind gift from Prof. Ying Jin [16] was grown on MEF (Mouse embryonic fibroblast) in human ESC culture medium comprised of Knockout-DMEM (Gibco) supplemented with $20 \%$ serum replacement (Invitrogen), $1 \mathrm{mM}$ glutamine, $0.1 \mathrm{mM}$ $\beta$-mercaptoethanol, $0.1 \mathrm{mM}$ non-essential amino acids, $50 \mathrm{U} / \mathrm{ml}$ penicillin plus $50 \mu \mathrm{g} / \mathrm{ml}$ streptomycin and $4 \mathrm{ng} / \mathrm{ml} \mathrm{bFGF}$ (Invitrogen). 


\section{Generation of IF and 2F AiPSCs from human AFSCs}

For lentivirus production, cDNAs encoding human OCT4 and SOX2 in EF1 vector containing a GFP report gene (a kind gift from Prof. Lei Xiao [17], Shanghai Institutes for Biological Sciences, SIBS) were co-transfected with packaging-defective helper plasmids into 293T cells using the LipoD293II (SignaGen, Gaithersburg, MD). Virus-containing supernatants were collected $48 \mathrm{~h}$ after transfection. The virus titer was adjusted to the ratio of ten virus liting a cell, which was judged by flow cytometry. For viral transduction, $2 \times 10^{4}$ human AFSCs were seeded on a 6-well plate. After the cells were evenly distributed on the culture dishes, OCT4 or OCT4 \& SOX2 viruses was added into the medium with polybrene for infection overnight. $24 \mathrm{~h}$ later, the medium was changed with fresh AFSC culture medium and after three days the cells were subjected to a second round of infection. The infection efficiency was judged by the percentage of GFP-positive cells using flow cytometry. Using this protocol, almost $100 \%$ infection efficiency was obtained. The second-round infected human AFSCs were cultured on $60 \mathrm{~mm}$ culture dish in AFSCs medium without mouse embryonic fibroblast (MEF) cells. After approximately 2 weeks, the cells were reduced in size, and assumed a round shape with cluster-like growth, at which point they were plated onto MEFs in a $100 \mathrm{~mm}$ culture dish in human ESC culture medium for 2-3 weeks. Upon the appearance of large colonies with typical ESC cell morphology (a high nucleus-to-cytoplasm ratio), colonies were mechanically divided and re-plated several times on to new feeder layers.

\section{Flow cytometry}

Surface markers were stained by incubating cells with each corresponding antibody for 30 min in dark according to the product instructions. Each test included an isotype-matched negative control. Cells were then washed twice with PBS containing 1\% FBS and resuspended in $0.5 \mathrm{ml}$ PBS. Flow cytometry was performed on a FACScan flow cytometer (Beckman Coulter, CA). Mouse monoclonal antibodies were obtained from the sources indicated: CD73, CD29, SSEA-3, SSEA-4, Tra-1-60 and Tra-1-81 (eBioscience, San Diego, CA); CD90, CD105, CD106 and CD45 (Biolegend, San Diego, CA); CD34 (BD Pharmingen, San Diego, CA); CD133 (Miltenyi Biotech). Primary antibodies were diluted to the concentration recommended by the supplier (generally $1 \mu \mathrm{g} / \mathrm{ml}$ ).

\section{RT-PCR analysis}

Total RNA was extracted from cells using a Mini
RNeasy Kit (QIAGEN GmbH) according to the manufacturer's instructions. For reversetranscription-polymerase chain reaction (RT-PCR), ThermoScript RT-PCR (Invitrogen) was used to synthesize cDNA from $1 \mu \mathrm{g}$ total RNA. PCR reactions were carried out by mixing $1 \mu \mathrm{l}$ of cDNA template, $250 \mathrm{nM}$ of each primer, $200 \mu \mathrm{M}$ dNTP mixture, and 1 $\mathrm{U}$ of Taq DNA polymerase in a volume of $20 \mu \mathrm{l}$. Samples were amplified in a thermocycler using the house keeping gene GAPDH as an internal standard. Primer information is offered (Supplementary Table S1). For expression of markers in human AFSCs, RT-PCR was performed on five human AFSC cell lines and a human ESC cell line (positive control), using primers against OCT-4, SOX2, NANOG, KLF4, C-MYC, REX1, and LIN28. For characterization of human AiPSCs cells, RT-PCR assays of AFSCs (negative control), 1F AiPSCs, 2F AiPSCs and human ESC (positive control), were performed using primers against endogenous OCT4, endogenous SOX2, NANOG, KLF4, C-MYC, REX1 and LIN28.

\section{Immunofluorescence Staining}

Cells grown in 8 well glass chamber slides (Nalgene Nunc, International) were washed twice in PBS and fixed in $4 \%$ paraformaldehyde for $30 \mathrm{~min}$, followed by permeabilization with $0.1 \%$ Triton-X-100 in PBS for $5 \mathrm{~min}$ at room temperature (RT). Cells were blocked for $30 \mathrm{~min}$ in 3\% BSA in PBS. All primary antibodies were diluted in the same blocking buffer and incubated with samples overnight at $4^{\circ} \mathrm{C}$. Primary antibodies were obtained from the sources indicated: OCT4, SOX2 and NANOG (Santa Cruz, Santa Cruz, CA); SSEA-3, SSEA-4, Tra-1-60 and Tra-1-81 (eBioscience, San Diego, CA).Cells treated with fluorescently coupled secondary antibody (anti-rabbit Cy3, Jackson ImmunoResearch, 1/200) were incubated for 1 hour at RT. Nuclei were counterstained with 4,6-diamindino-2-phenylindole2- (DAPI) (Sigma) for $3 \mathrm{~min}$ at RT. Preparations were viewed under a BX-60 epifluorescence micro-scope (Olympus, China), and images were captured with an Axiocam digital camera (Carl Zeiss, Jena, Germany, http://www.zeiss.com) and analyzed using KS-300 software (Imaging Associates, Thame, China).

\section{Cytogenetic Analysis}

A minimum of 20 metaphases were examined by the Reproductive Biology and Genetic Centre of the City Hospital of Suzhou. The karyotypes were captured and documented using Apply Imaging System for cytogenetic analysis.

\section{Microarray and DNA methylation analysis}

Affymetrix human U133 plus 2.0 array chips were used for analysis of global gene expression. For 
global gene expression analysis in human AFSCs (negative control), 1F human AiPSCs, 2F human AiPSCs, human ES cells (positive control), three samples for each type of cells and three technical replicates for each sample were analyzed in the microarray assay. The Sequenom EpiTYPER method was used to analyzing DNA demethylation patterns of the OCT4, SOX2 and NANOG gene promoter regions. Promoter region information is provided (Supplementary Table S2). Both experiments were performed and analyzed at the BeiJing CapitalBio Corporation. Samples of three biological repeats from human AFSCs, $1 F$ \& 2F human AiPSCs and a human ESC line (SHhES2), were collected and analyzed as described in the Affymetrix Technical and Sequenom EpiTYPER Manual.

\section{Isolation and biological activity analysis of MVs}

Culture media were collected every $48 \mathrm{~h}$ and centrifuged at $3,000 \times \mathrm{g}$ for $30 \mathrm{~min}$ to remove cellular debris. Supernatants were centrifuged at $100,000 \times g$ for $2 \mathrm{~h}$. Pelleted AiPSC-MVs and Fib-MVs were washed in cold PBS supplemented with 5m MHEPES and their concentration estimated by Bradford assay. To test the biological activity of the AiPSC-MVs, cells were seeded on cover slips and nuclei were stained with DAPI (Sigma) by adding dye to culture medium for $5 \mathrm{~min}$. AiPSC-MVs were stained with $4 \times 10^{-7 \mathrm{M}}$ PKH26 Red Fluorescent Cell Linker Mini Kit for General Cell Membrane Labeling (Sigma) after which they were neutralized by the addition of serum, washed with PBS and added to the culture medium. Cells were incubated with PKH26 stained MVs for 5 min, $30 \mathrm{~min}, 5 \mathrm{~h}$, and 10 hours or with PKH26 only. After washing with PBS the cover slips were mounted.

\section{Teratoma formation}

For teratoma induction assay, $1 \times 10^{6}$ cells from each human AiPSC cell line were harvested and mixed with matrigel and injected intramuscularly into 8 NOD-SCID mice (from the Chinese Academy of Sciences, China). For negative control experiments, matrigel containing medium was injected intramuscularly into 3 NOD-SCID mice. All mice were treated using ethyl ether for 30 seconds before injection. After 2 month, teratomas were stained with H\&E (hematoxylin and eosin).

\section{Model of Acute Ischemic Injury}

For Acute Ischemic Injury model, Healthy male Wistar rats, weighing 250-300 g and aged 3-4 months ( from the Chinese Academy of Sciences, China) were subjected to transient middle cerebral artery occlusion (MCAO). For Anesthesia of rats, chloral hydrate was intraperitoneally injected with the proportion of
$300 \mathrm{mg} / \mathrm{kg}$ (chloral hydrate/rat weight). The middle cerebral artery was occluded using a nylon suture filament $(0.24 \mathrm{~mm})$ for $2 \mathrm{~h}$. Reperfusion was performed by removing the suture. Out of 72 rats subjected to focal cerebral ischemia, 16 died and 12 did not exhibit paralysis of the limbs. 4 rats out of 16 died were due to over anesthesia and the other 12 rats died because of Arterial embolism operation. The remaining rats were randomly divided into three groups ( $\mathrm{n}=10$ per group). For MVs injection, one week after MCAO, the rats were fixed on a stereotactic apparatus and the bregma was opened by a head scalp incision. Coordinates were marked $(1 \mathrm{~mm}$ anterior to the skull, left margin $2.5 \mathrm{~mm}$; depth 4.5-5.5 $\mathrm{mm}$ ), and a $5 \mu \mathrm{l}$ AiPSC-MVs suspension, PBS or Fib-MVs control was then injected into the striatum using a Hamilton syringe for 10 min. 5 days after MV injection, the brains were quickly extracted. A $\sim 2 \mathrm{~cm}$ ischemic area of brain tissue was excised and post-fixed in $4 \%$ paraformaldehyde. 5- $\mu \mathrm{m}$ coronal brain slices were consecutively sampled by paraffin sections. Out of 480 slices, every 20th slice was stained to find the needle position.

\section{Neurological behavior evaluation}

Neurological behavior evaluations were measured before MVs injection and 1, 2, 3 weeks after MVs injection by the neurological severity score (NSS; Supplementary Table S3), beam balance test (BBT; Supplementary Table S4) and elevated body swing test (EBST). In EBST study, observers were from the vertical axis within 30 second of the raising rats by the tail. The experiment was followed by 1 min of rest, and the test was repeated 20 times. All rats were tested three times at different time-points and the average score was tested. (iPS-MVs treatment group $=5$, PBS- treatment group $=5$, Fib-MVs treatment group $=5$ )

\section{Statistical analysis}

Data were presented as the mean \pm standard deviation. Comparisons of neurological scores were carried out by ANOVA (F test, q test), using SPSS 10.0. The paired t-test was used for the cell count. A value of $P<0.05$ indicated a statistically significant result.

\section{Ethics Statement}

In this study, human AFSCs samples collection with written consent of subjects and the written approval from the ethical review board of the first affiliated hospital in Soochow University and the city hospital of Suzhou. Duplicated written consent of subjects along written approval was kept in the hospital ethical review board office. All experimental procedure using human AFSCs samples in this study was reviewed and approved by the ethics committee. 
Mice and rats are used in the present study; both were handled in strict accordance with good animal practice and all animal work. All experimental procedure of using mice and rats in this study was reviewed and was approved by the ethical review board of the first affiliated hospital of Soochow University.

\section{Results}

\section{Derivation and characterization of human AFSCs}

We firstly used flow cytometry to assess evaluate the expression of ESC and adult stem cell markers in human AFSCs (Fig. 1A). All 17 human AFSCs cell lines gave similar results. AFSCs were negative for markers of the hematopoietic lineage (CD45), endothelial cells (CD106) and hematopoietic stem cells (CD34, CD133), but were positive for surface markers characteristic of mesenchymal and/or neural stem cells, including CD29 (hyaluronan receptor), CD73, CD90 and CD105 (endoglin). Human AFSCs also were positive for stage-specific embryonic antigen (SSEA)-4, a marker expressed by ESCs, but were negative for SSEA-3, Tra-1-60 and Tra-1-81. These results indicated that human AFSCs have characteristics of both ESCs and adult stem cells. To evaluate the feasibility of producing $1 \mathrm{~F}$ and $2 \mathrm{~F}$ AiPSCs, we next compared the expression in human AFSCs and human ESCs of several ESC marker genes used in iPSCs reprogramming (Fig. 1B). RT-PCR analysis showed that AFSCs exhibited strong expression of MYC and Rex-1, moderate expression of OCT4, NANOG, and KLF4 and sporadic expression of SOX2, but did not express LIN28. These data indicated to us that human AFSCs expressed most iPSC reprogramming factors, and suggested that they could theoretically be reprogrammed into iPSCs using a low number of such factors.

\section{Generation of IF and 2F AiPSCs from human AFSCs}

Next, human AFSCs $(\sim 20,000$ cells $)$ in AFSCs culture medium were infected with EF1 lentiviral vector containing either human OCT4 CDNA, or a 1:1 mixture of human OCT4 and SOX2 cDNAs for OCT4 and SOX2. Using this protocol, almost $100 \%$ infection efficiency was obtained. Of three SOX2 positive AFSC cell lines tested, one was a 1F AiPSC cell line and the other two were 2F AiPSC cell lines. A total of four 1F AiPSC clones out of seven human ESC-like colonies were obtained by selecting from OCT4-infected human AFSCs (reprogramming efficiency $=0.0066 \%$ ) and a total of $182 \mathrm{~F}$ AiPSC clones were selected from ESC-like colonies in OCT4 and SOX2-infected human
AFSCs (reprogramming efficiency $=0.03 \%$ ).

To determine whether prolonged culturing would induce pluripotency in human AFSCs, untransfected human AFSCs were cultured on MEFs in human ESC medium for more than six weeks. After this period, most AFSCs had differentiated into fibroblast-like cells, without any ESC-like colonies.

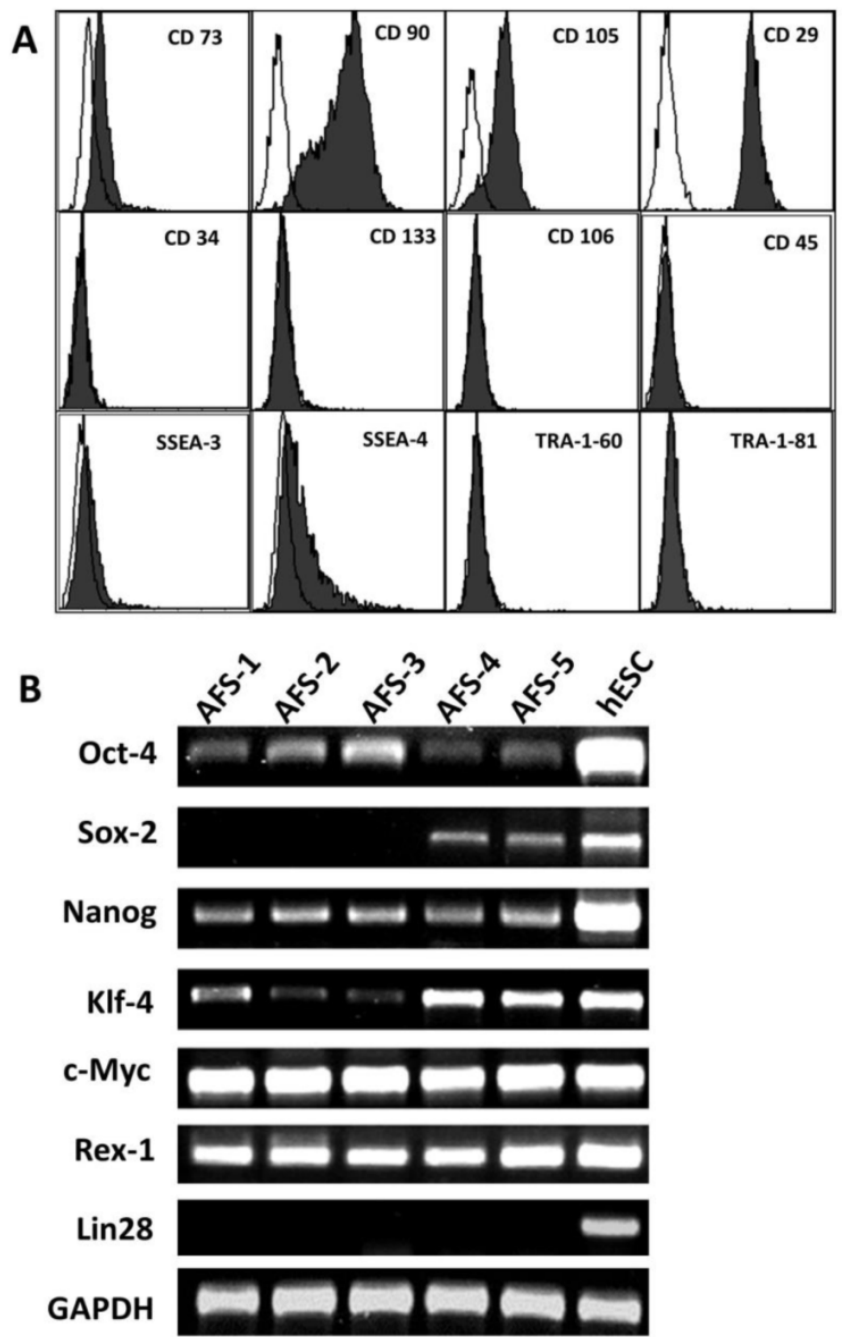

Figure 1. Expression of markers in human AFSCs. (A) Expression of surface antigens was carried out by flow cytometry using mouse monoclonal antibodies (filled curve). All panels include an isotype-matched negative control (unfilled curve). Antigens tested were, as indicated on panels: CD73, CD90, CD105, CD29, CD34, CD133, CD106, CD45, SSEA-3, SSEA-4, TRA-1-60 and TRA-1-81. (B) RT-PCR was performed on five human AFSC cell lines and a human ESC cell line (positive control), using primers against OCT-4, SOX2, NANOG, KLF4, C-MYC, REXI, and LIN28.

\section{Characterization of human AFSCs derived-iPSCs (human AiPSCs) cells}

Of the 22 human ESC-like colonies, four were expanded over 35 passages for further characterization. RT-PCR analysis (Fig. 2G) showed that relative to parental AFSCs, $1 \mathrm{~F}$ and $2 \mathrm{~F}$ human AiPSCs cells expressed increased levels of OCT4, 
SOX2, NANOG, KLF4 and LIN28, and comparable levels of MYC and REX1. Most human ESC markers expression level of $1 \mathrm{~F}$ and $2 \mathrm{~F}$ human AiPSCs cells were similar to them in human ESC. Immunofluorescence staining (Fig. 2H--N) showed that $1 \mathrm{~F}$ AiPSCs expressed OCT4, SOX2 and NANOG transcription factors and the human ESC stage-specific surface markers SSEA3, SSEA4, TRA-1-60 and TRA-1-81. For evaluation of chromosomal abnormalities of human AiPSCs cells, cytogenetic analysis was performed using metaphase spreading. $1 \mathrm{~F}$ and $2 \mathrm{~F}$ human AiPSCs (AFS derived iPS) cells kept normal karyotypes during reprogramming. To exclude the possibility that iPSCs cell clones arose from human ES cell contamination, the genotypes of human AFSCs $(46 \mathrm{XX})$ and AiPSCs $(46 \mathrm{XX})$ and SHhES2 cells (46 XY) were confirmed, further verifying the identity of our AiPSCs cells (Fig. 2F).
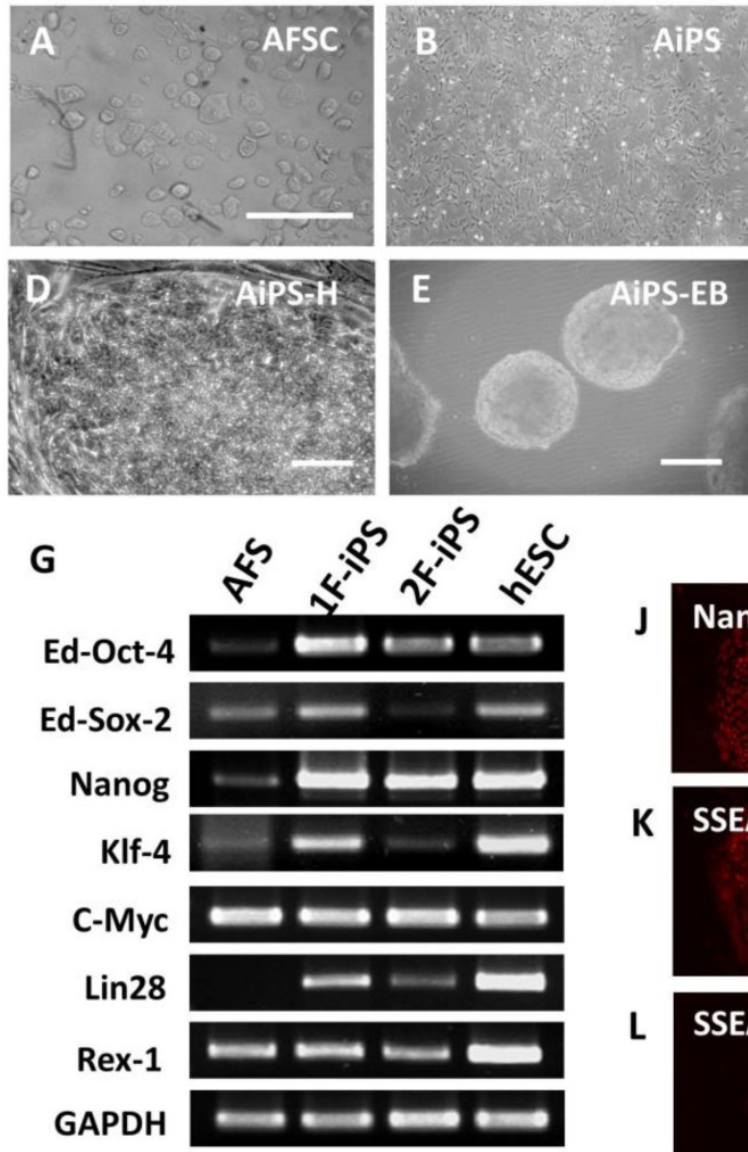

H
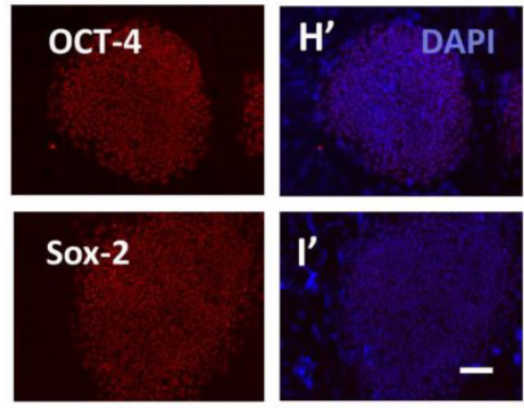

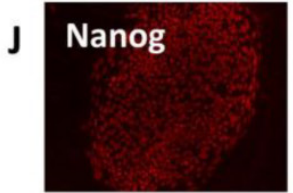

K
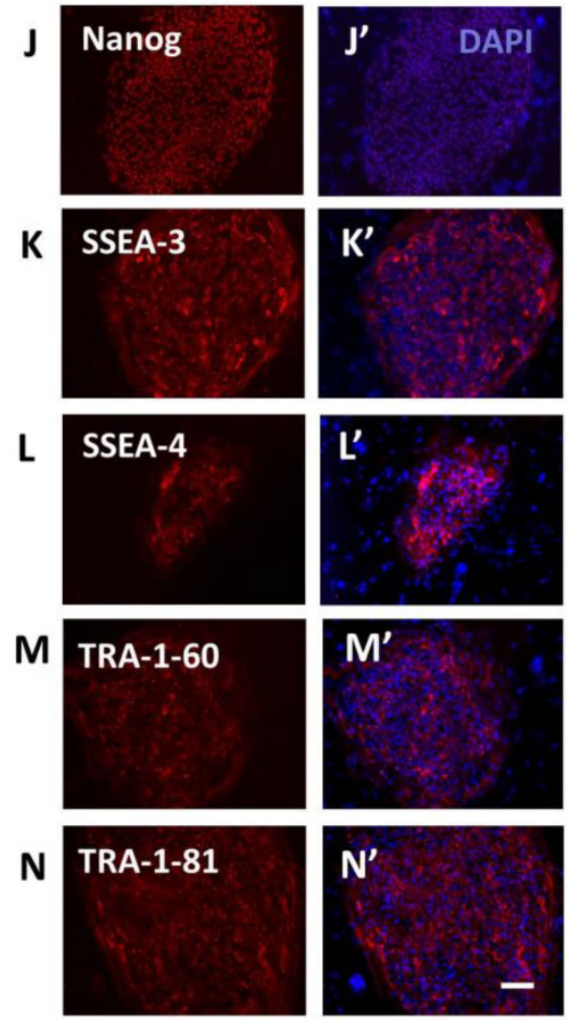

Figure 2. Derivation and characterization of human AiPSCs cells. (A-E) Morphology of amniotic fluid cells at low magnification (D); CD-117 positive AFSCs at low magnification (B)Typical undifferentiated human ESC-like colonies of human OCT4 IF AiPSCs at low (C) and high (D) magnification in culture dishes after six weeks post viral infection; human IF AiPSCs form a suspended embryo body(E) and the genotype of human IF AiPSCs (46 XX) is identical to human AFSCs (46 XX) (F) All scale bars are $250 \mu \mathrm{m}$. (G) RT-PCR assays of AFSCs (negative control), $1 \mathrm{~F} \mathrm{AiPSCs,} 2 \mathrm{~F}$ AiPSCs and human ESC (positive control), were performed using primers against endogenous OCT4, endogenous SOX2, NANOG, KLF4, C-MYC, REXI and LIN28. (H-N) Immunocytochemical analysis of pluripotency and surface markers (OCT4,

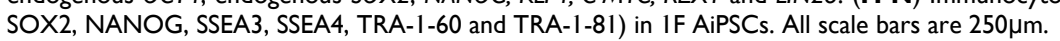



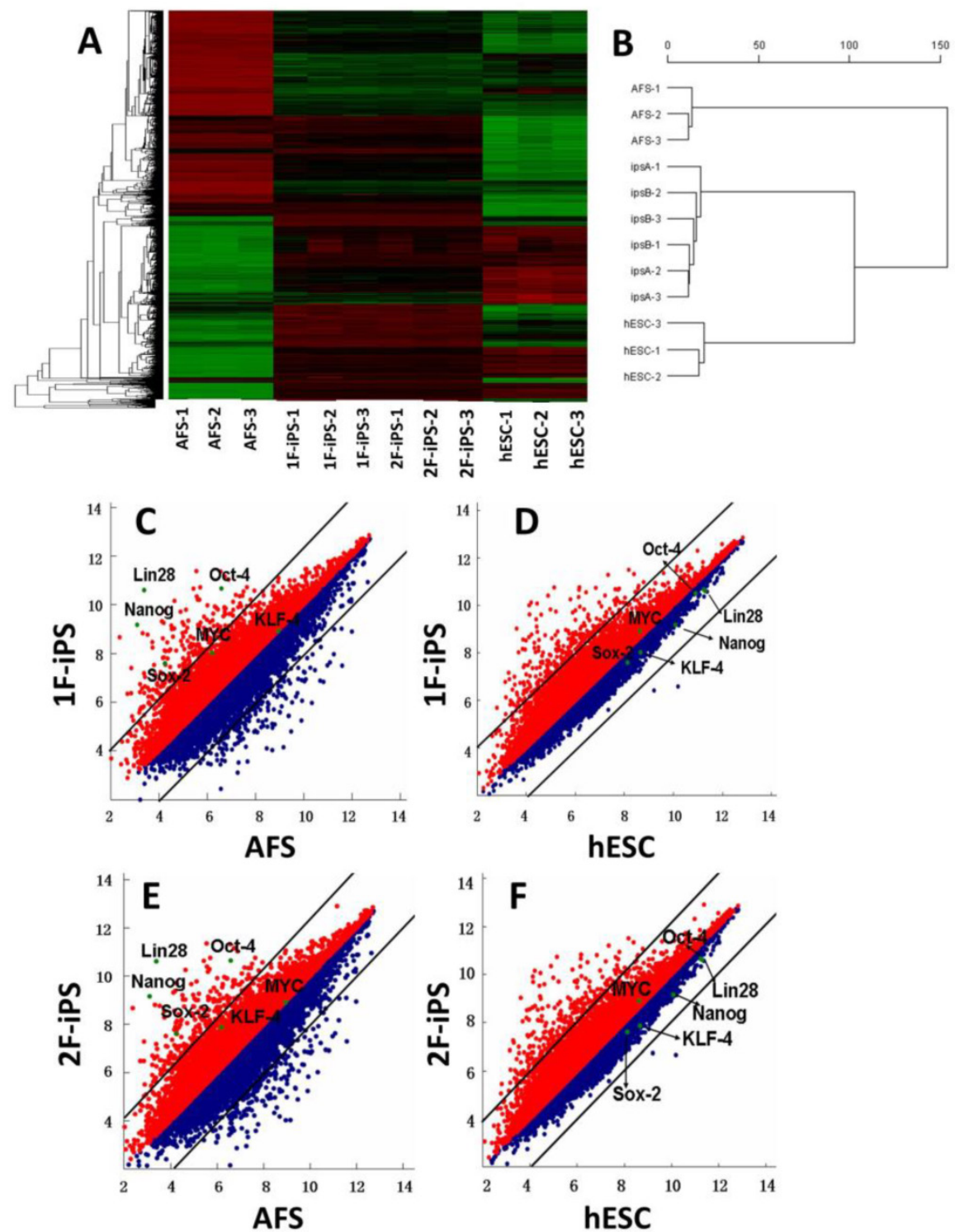

Figure 3. Global gene expression profiling and DNA methylation analysis of human AFSC-derived iPSCs. (A) Heat map analysis (left) and hierarchical cluster analysis (right) of gene expression from human AFSCs, 1F human AiPSCs, 2F human AiPSCs, human ES cells. (C-F) Scatter plots comparison of global gene expression profiles between IF human AiPSCs cells and human AFSCs (C), 1F human AiPSCs cells and human ES cells (D), 2F human AiPSCs cells and human AFSCs (E), and 2F human AiPSCs cells and human ES cells (F).

\section{Global gene expression analysis and DNA methylation analysis in human AiPSCs}

We next performed global gene expression analysis on human AFSCs, $1 \mathrm{~F}$ and $2 \mathrm{~F}$ human AiPSCs and human ESCs. Heat mapping (Fig. 3A, left panel), hierarchical clustering (Fig. 3B, right panel) and scatter plot analysis (Fig. 3C-F) indicated that the global expression profiles of $1 \mathrm{~F}$ and $2 \mathrm{~F}$ human AiPSCs cell were similar to those in human ESCs, but distinct from the parental AFSCs. Based upon these results, we concluded that human AFSCs were successfully reprogrammed into AiPSCs by OCT4 alone, or by OCT4 and SOX2.
We next used Sequenom EpiTYPER analysis to compare the DNA methylation patterns of the OCT4, SOX2 and NANOG promoters between the different cell lines (Fig. 4). We found that compared to AFCSs, $1 \mathrm{~F}$ and $2 \mathrm{~F}$ human AiPSCs cells and ESCs had lower levels of methylation in the promoter regions of all three genes. Unexpectedly, although SOX2 expression was weak or negative in AFCSs, we found that the extent of demethylation of the SOX2 promoter in AFSCs was higher than in human ESCs, a result we confirmed using primers to amplify other sequences in the SOX2 promoter region. It is conceivable that in these cells, although the SOX2 promoter region is transcriptionally competent, other mechanisms are acting to restrict expression of SOX2. 

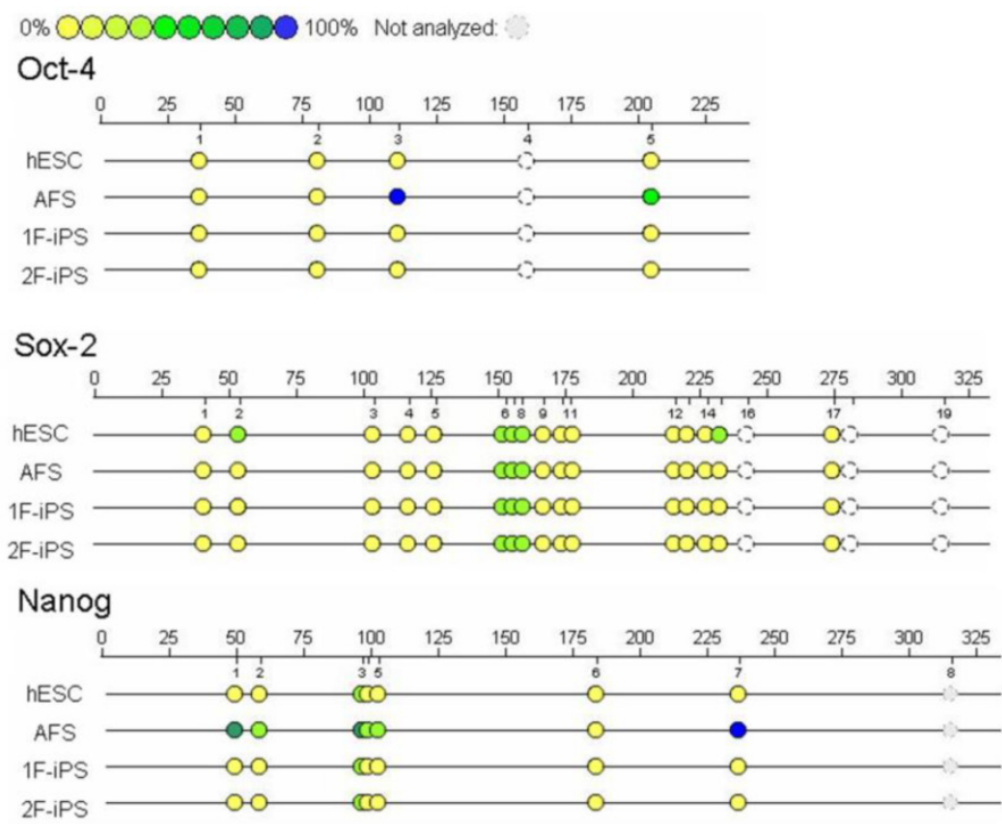

Figure 4. Sequenom of human AFSC-derived iPSCs. Sequenom EpiTYPER analysis was performed to determine the degree of DNA methylation of the OCT4, SOX2 and NANOG promoters. Yellow circles represent unmethylated $C_{p} G$ islands, while blue circles represent methylated $C_{p G}$ islands.

\section{In vitro and in vivo pluripotency of human AiPSCs}

In vitro pluripotency of $1 \mathrm{~F}$ and $2 \mathrm{~F}$ human AiPSCs cells was tested by embryoid body (EB) formation (Fig. 2E). During EB period, human AiPSCs cells were easy to form globular structure with a variety of cell types. RT-PCR analysis confirmed the expression in AiSPCs of markers for endoderm (PAX6 and AFP), mesoderm (ANP and Brachyury), ectoderm (Nestin) and trophectoderm $(\mathrm{Cd} \times 2)$ (Fig. 5A). We next evaluated the in vivo pluripotency of AiPSCs cells by subcutaneous transplantation into NOD-SCID mice. Two month after injection, all 8 NOD-SCID mice injected with $1 \mathrm{~F}$ and $2 \mathrm{~F}$ human AiPSCs cells grew teratomas and three negative control mice grew without teratomas. During two mouths, the mice with teratomas became thin due to tumor and the negative control mice seem normal health. All mice did not exhibit signs of pain/distress. The Size of all teratomas was around $5 \times 8 \mathrm{~mm}$. The derivatives of all three germ layers were found in the teratomas formed from $1 \mathrm{~F}$ and $2 \mathrm{~F}$ human AiPSCs cells, including endoderm (intestinal epithelium), mesoderm (cartilage) and ectoderm (neural epithelium, keratinized stratified squamous epithelium). (Fig.5D-G). To exclude the possibility of pluripotent cells existing in the AFSCs, a teratoma formation assay was carried out for human AFSCs and there was no evidence of teratoma in any samples after long term observation. These results indicated that like human ES cells, 1F and 2F human AiPSCs cells were pluripotent in vitro and in vivo.

\section{AiPSC-MVs have biological activity and can enter cells}

We next evaluated the biological activity of AiPSC-MVs. We observed characteristic PKH26 staining in AFSCs exposed to PKH26-labeled AiPSC-MVs (Figure 6a-d), but not PKH26 alone (Figure 6e), indicating that AiPSCs acted as 'physiological liposomes' by attaching to and fusing with target cells, and transferring their contents into the cytoplasm.

\section{AiPSC-MVs mediate repair of acute ischemic injury}

Next, we assessed the response in a rat model of brain injury after ischemia to injection of AiPSC-MVs. We found that compared with the infarct volume in PBS $\left(30.8 \pm 3.1 \mathrm{~mm}^{3}\right)$ or Fib-MVs injected animals $(27.2$ $\left.\pm 1.1 \mathrm{~mm}^{3}\right)$, infarct volume in the AiPSC-MVs treated group $\left(13.7 \pm 1.4 \mathrm{~mm}^{3}\right)$ was reduced $(p<0.05$; Figs. 6B and C), indicating that AiPSC-MVs mediated repair of ischemic injury.

\section{Neurological function recovery of AiPSC-MVs treated rats}

AiPSC-MVs, PBS and Fib-MVs treated rats were measured for neurological function using the NSS, BBT and EBST tests (Fig. 7A-C) after injection 0, 1, 2 and 3 weeks. In the study, the neurological deficit of AiPSC-MVs treated rats were markedly improved, indicating that AiPSC-MVs promoted neurological function recovery of Acute Ischemic Injury rats. 

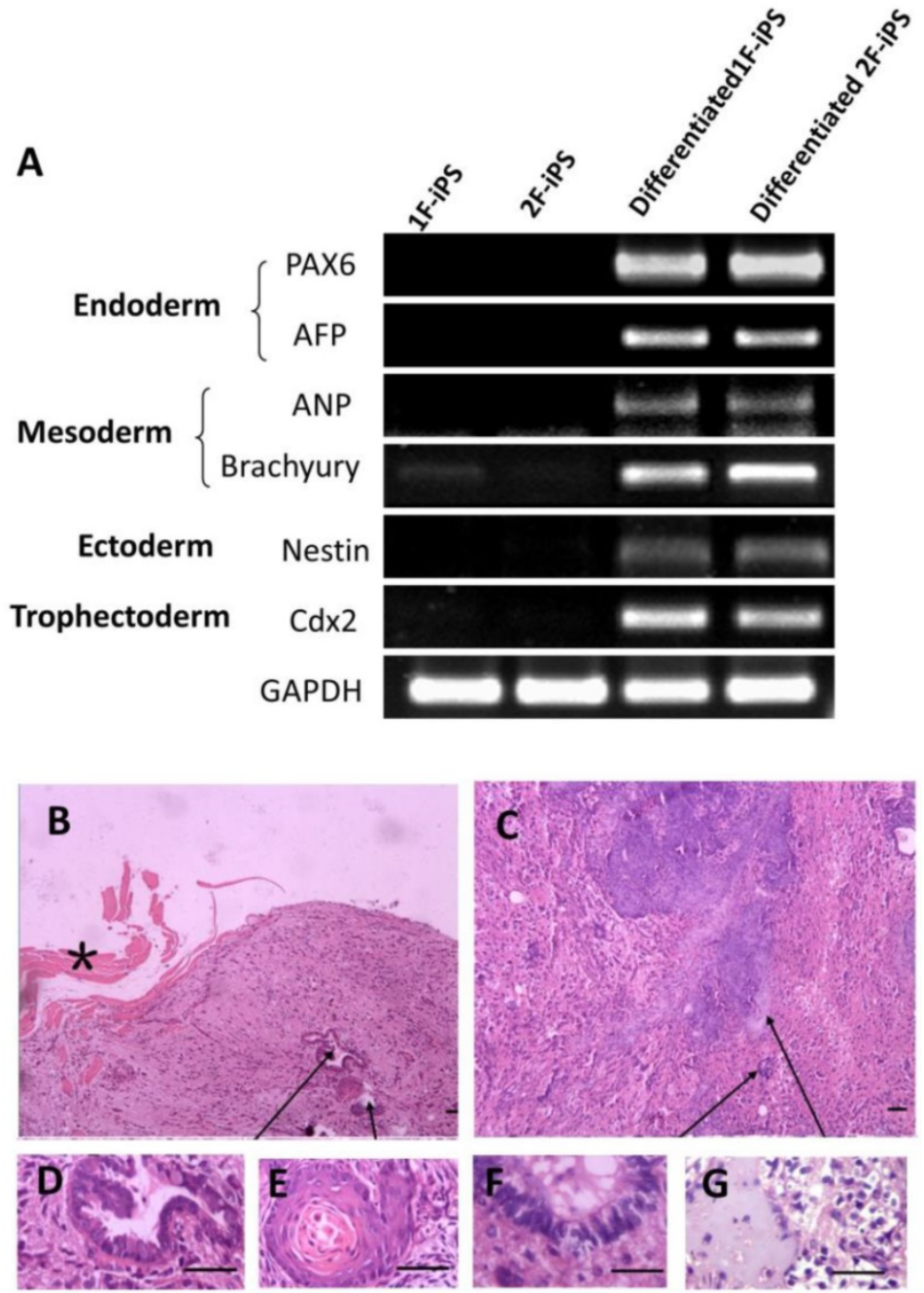

Figure 5. Differentiation of human AiPSC cells In vitro and in vivo. (A) RT-PCR analysis of expression levels of three germ layer and trophoectoderm markers was performed for embryo body samples of $1 \mathrm{~F}$ human AiPSCs and 2F human AiPSCs. (B-C) Teratomas were sectioned and stained with hematoxylin and eosin. Histological section of identified cells representing all three germ layers: endoderm (intestinal epithelium, D), mesoderm (cartilage, G) and ectoderm (neural epithelium, F; keratinized stratified squamous epithelium, E). Enlargements of sections showing tissues are indicated by arrows, *shows the mouse skeletal muscle, Scale bars, $100 \mu \mathrm{m}$.

\section{Discussion}

This study demonstrates that ectopic expression of OCT4 alone is sufficient to directly reprogram AFSCs to pluripotent $1 \mathrm{~F}$ AiPSCs. The expression profiles of selected pluripotency markers and transcription factors in 1F AiPSCs was similar to that in ESCs, as was the extent of demethylation in the OCT4, SOX2 and NANOG promoter regions. Moreover, the majority of $1 \mathrm{~F}$ AiPSCs cells maintained normal karyotypes, even after 35 passages, and efficiently differentiated into all three germ layer cells, as well as forming teratomas in vivo.

AFSCs express high levels of SOX2, NANOG, MYC, KLF4 and low levels of OCT4. Accordingly, expression of OCT4 in AFSCs such as NSCs (neuron stem cells) would be theoretically sufficient for successful iPSCS reprogramming. The vertebrate neural crest is a pluripotent embryonic cell population derived from the lateral ridges of the neural plate during closure of the neural tube. Neural crest cells disperse from the dorsal surface of the neural tube and migrate extensively through the embryo, giving rise to a wide variety of differentiated cell types. During this migration stage, NSCs may remain in the amniotic cavity to form a small stem cell population in amniotic fluid [18]. Furthermore, amniotic fluid contains a sub-population of NESTIN positive cells $(<10 \%)$, and a much smaller AF sub-population of CD117 positive cells $(<1 \%)$ which express pluripotency markers. SOX2-expressing AF clones have been shown to give rise to a neuron-like phenotype in culture, expressing neuronal markers such as MAP2, NFL and NSE [19]. Taken together, these data indicate that a small NSC like population $(<10 \%)$ is originated from CD117 positive AFSCs derived iPS cells. 
A
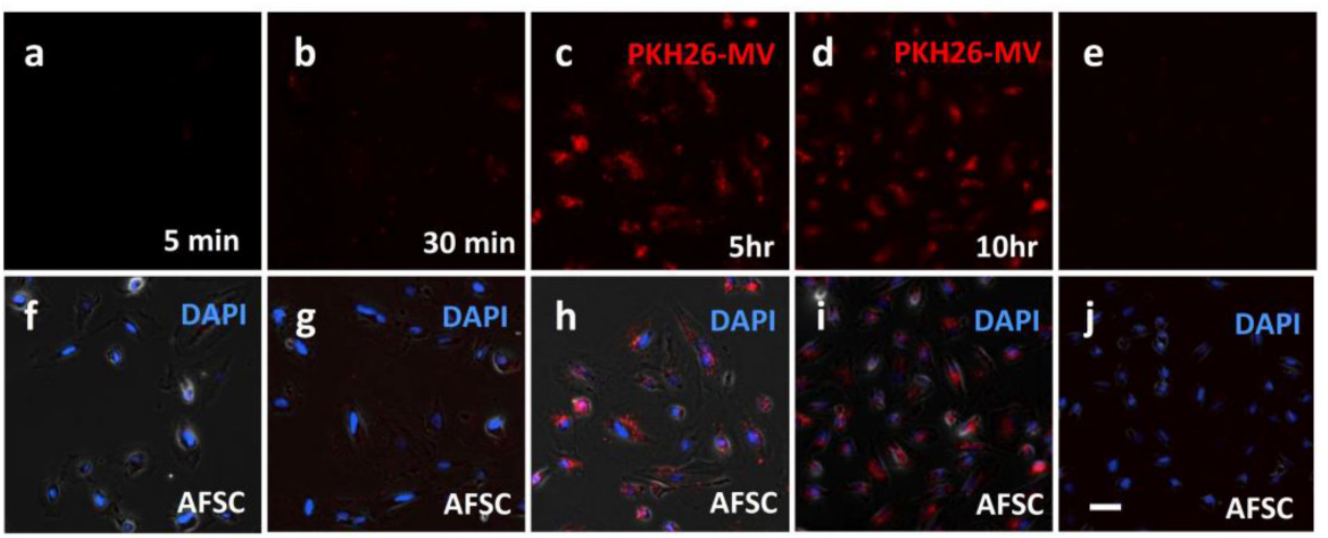

B

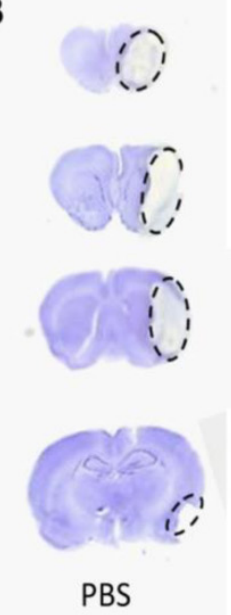

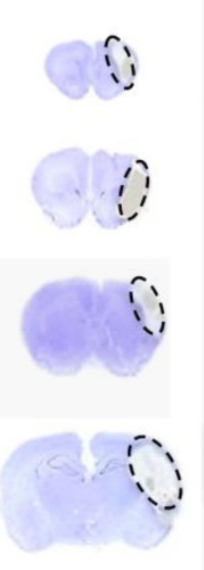

Fib-MV

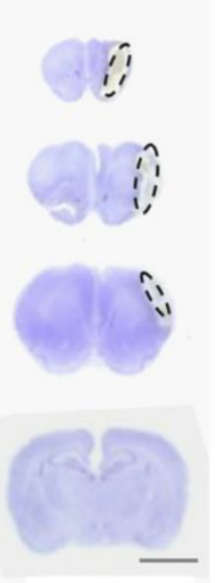

iPS-MV
C

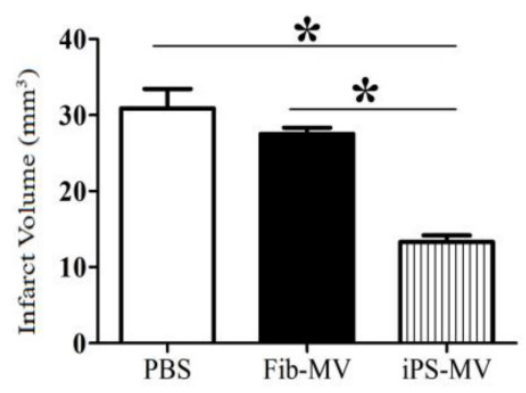

Figure 6. Regeneration potential of AiPSC-MVs on cerebral ischemia injury. (A) AiPSC-MVs enter target cells. Human AFSCs were exposed to AiPSC-MVs labeled with 4x10-7M PKH26 (red) for 5 min(a), 30 min (b), 5 hours (c), 10 hours (d) or PKH26 only (e), Nuclei of human AFSCs stained with DAPI (blue) and both colors merged (f-j). (B) Nissl staining indicates that AiPSC-MVs treated rat have much smaller infarct areas than those of PBS and Fibroblast-MV control animals. (C) Quantitative analysis shows that systemic administration of AiPSC-MVs significantly reduced the infarct volume three days after transient MCAO compared with control animals. Data are mean SD, $n=10$ in each group. ${ }^{*} \mathrm{p}<0.05$, AiPSC-MVs treated mice versus PBS or Fibroblast-MV treated rats.

A
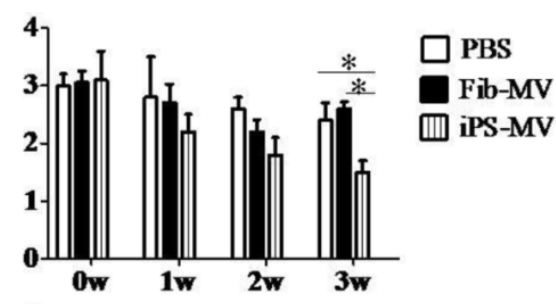

B
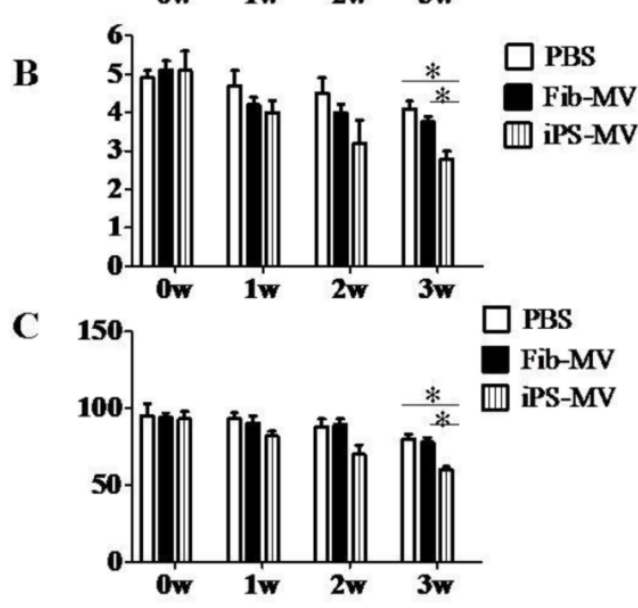

Figure 7. AiPS-MVs promoting neurological deficit recovery. Comparison of Score in (A) NSS, (B) BBT and (C) EBST of AiPS-MVs, PBS or Fibroblast-MV treated group. The neurological behaviors of the brain ischemic rats treated by IF AiPS-MVs were greatly improved. NSS, neurological severity score; BBT, balance beam test; EBST, elevated body swing test.

Limiting the number of factors for generating iPSCs is of great significance, both to reduce the number of oncogenes such as MYC and KLF4 [3,5], which can give rise to tumors in offspring, and to minimize the probability of retroviral insertional mutagenesis. Analysis of previously reported iPSCs show up to 20 retroviral genomic integrations of external transgene $[3,20]$. Limiting the number of factors is also important because, due to the number of exogenous factors currently required, the molecular mechanisms for reprogramming are not completely understood. Alternative approaches to generating human iPSCs other than viral transduction of transcription factors include direct delivery of 
recombinant proteins [21,22] and small molecules [23]. Incorporating such methodologies into our $1 \mathrm{~F}$ AiPSCs system would offer a new approach to understanding the molecular mechanisms of iPSC reprogramming by quantitative microinjection of a single transcription factor.

Previous reports of one-factor generation of iPSCs include the use of OCT4 to reprogram NSCs of mouse [6] and human [7]. Human NSCs are typically isolated from limited resource of fetal brain tissue, which limits the clinical application of one-factor iPSCs from human NSCs. In our AiPSC system, the donor AFSCs were isolated from amniotic fluid which the supply source is abandoned supply in clinic, and unencumbered by ethical issues. AFSCs were resourceful and easy to build the cell bank for storage. AFSCs therefore have great potential to generate patient-specific AiPSCs for application in autologous stem cell treatment in the later development of the baby, as well as for infants in whom a genetic disorder has been detected by amniocentesis.

We also demonstrated the potential of AiPSC-MVs in promoting neural regeneration and repairing acute ischemic injury. Since AiPSCs theoretically possess the memory characteristics of NSCs in addition to ESC character, AiPSC-MVs may therefore contain signaling molecules that mediate both nerve repair and regeneration of stem cells. Ratajczak's group had shown the role and mechanism of soluble factors and MVs derived from stem cells employed as cellular therapeutics in regenerative medicine [24]. The potential mechanism by which human iPS-MV improved stroke in rats, is that iPS-MV may deliver RNA and microRNA with therapeutic effects into damaged brain. There were some species specific differences in mRNA and proteins between human and rat. However, human and rat still have the compatibility of bioactive lipids such as sphningosine-1 phosphate, ceramide-1 phosphate that are enriched in MVs between both species. Both S1P and $\mathrm{C} 1 \mathrm{P}$ have strong proangiopoietic and antiapoptotic effects. We prove the use of MVs isolated from tissue specific iPSCs able repair tissue damage without the use of iPSCs which represent a novel approach to overcoming one of the current bottlenecks in iPSC technology.

\section{Supplementary Material}

Tables S1-S4. http://www.ijbs.com/v12p0558s1.pdf

\section{Acknowledgement}

We thank Anthony Atala for the suggestions regarding the purification and culture of AFSCs; Prof. Lei Xiao for the gift of Lentiviral vector; Prof. Ying Jin for the gift of the human ESC line (ShhES2). This work was supported by The National Natural Science Foundation (31000654), Ministry of Education of New Funds for Teachers Grant, the 973 Plan Research Special Subject (2012CB22302) and a Project Funded by the Priority Academic Program Development of Jiangsu Higher Education Institutions (PAPD).

\section{Competing Interests}

The authors have declared that no competing interest exists.

\section{References}

1. Takahashi K, Yamanaka S. Induction of Pluripotent Stem Cells from Mouse Embryonic and Adult Fibroblast Cultures by Defined Factors. Cell. 2002; 126: 663-676.

2. Yu J, Vodyanik MA, Smuga-Otto K, et al. Induced pluripotent stem cell lines derived from human somatic cells. Science.2007; 318:1917-1920.

3. Wernig M, Meissner A, Jaenisch R, et al. In vitro reprogramming of fibroblasts into a pluripotent ES-cell-like state. Nature. 2007; 448:318-324.

4. Okita $\mathrm{K}$, Nakagawa $\mathrm{M}$, Hyenjong $\mathrm{H}$, et al. Generation of mouse induced pluripotent stem cells without viral vectors. Science. 2008; 322: 949-953.

5. Huangfu D, Maehr R, Guo W, et al. Induction of pluripotent stem cells by defined factors is greatly improved by small-molecule Compounds. Nat Biotechnol. 2008; 26:795-797.

6. Kim JB, Sebastiano V, Wu G, et al. Oct4-Induced pluripotency in adult neural stem cells. Cell.2009; 136: 411-419.

7. Kim JB, Greber B, Marcos J, et al. Direct reprogramming of human neural stem cells by OCT4. Nature.2009; 461:649-653.

8. Chunliang Li, Junmei Zhou, Guilai Shi, et al. Pluripotency can be rapidly and efficiently induced in human amniotic fluid-derived cells. Human Molecular Genetics. 2009; 18: 43-40.

9. Lin Ye, Judy C Chang, Chin Lin, et al. Induced pluripotent stem cells offer new approach to therapy in thalassemia and sickle cell anemia and option in prenatal diagnosis in genetic diseases. PNAS.2009; 24:9826-9830.

10. Coppi PD, Bartsch G, Atala A, et al. Isolation of amniotic stem cell lines with potential for therapy. Nat. Biotechnol.2007; 25:00-106.

11. M Carmen Martinez, Ramaroson Andriantsitohaina. Microparticles in Angiogenesis: Therapeutic Potential. Circulation Research.2011; 109:110-119.

12. Susmita Sahoo, Ekaterina Klychko, Raj Kishore, et al. Exosomes from human CD34 stem cells mediate their proangiogenic paracrine activity. Circulation Research.2011; 109:724-8.

13. Marcin Wysoczynski, Mariusz Z. Ratajczak. Lung cancer secreted microvesicles: Underappreciated modulators of microenvironment in expanding tumors. Int J Cancer. 2009;125:595-1603.

14. A J Wieczorek, A Kowalska, MariuszZ Ratajczak. Platelet-derived microparticles bind to hematopoietic stem/progenitor cells and enhance their engraftment. BLOOD. 2001; 98:3143-3149.

15. J Ratajczak, K Miekus, M Kucia, et al. Embryonic stem cell-derived microvesicles reprogram hematopoietic progenitors: evidence for horizontal transfer of mRNA and protein delivery. Leukemia.2006; 20:847-856.

16. Chunliang Li, Ying Yang, Xiaowei Lu, et al. Efficient derivation of Chinese human embryonic stem cell lines from frozen embryos. In Vitro Cellular \& Development. 2010; 46: 186-191.

17. Liao J, Cui C, Chen S, et al. Generation of Induced Pluripotent Stem Cell Lines from Adult Rat Cells. Cell Stem Cell.2008; 4:11-15.

18. Huszar D, Sharpe A, Jaenisch R. Migration and proliferation of cultured neural crest cells in W mutant neural crest chimeras. Development.1991; 112:131-141.

19. A Jezierski, M Sikorska, M Bani-Yaghoub, et al. Probing Stemness and Neural Commitment in Human Amniotic Fluid Cells. Stem Cell Rev and Rep. 2010; 6:199-214.

20. Aoi T, Yae K Nakagawa, M Ichisaka, et al. Generation of pluripotent stem cells from adult mouse liver and stomach cells. Science.2008; 321:699-702.

21. Hongyan Zhou, Shili $\mathrm{Wu}$, Jin Young, et al. Generation of induced pluripotent stem cells using recombinant proteins. Cell Stem Cell.2009; 4:381-4

22. Dohoon Kim, Chun Hyung, Kim Kwang-Soo, et al. Generation of human induced pluripotent stem cells by direct delivery of reprogramming proteins. Cell Stem Cell.2009; 4:472-6.

23. Justin K, et al. A small-molecule inhibitor of TGF- $\beta$ signaling replaces Sox 2 in reprogramming by inducing Nanog. Cell Stem Cell.2009; 5:491-503.

24. Ratajczak MZ, Kucia M, Jadczyk T, et al. Pivotal role of paracrine effects in stem cell therapies in regenerative medicine: can we translate stem cell-secreted paracrine factors and microvesicles into better therapeutic strategies. Leukemia.2012; 26:1166-73. 